Punjab University Journal of Mathematics (2021),53(7),497-509

https://doi.org/10.52280/pujm.2021.530704

\title{
An Efficient Two-Step Iterative Technique for Solving Non-Linear Equations
}

\author{
Muhammad Shazib Hameed ${ }^{* 1}$, Zaheer Ahmad ${ }^{2}$, Faisal Ali ${ }^{3}$ \\ 1,2 Department of Mathematics, Khwaja Fareed University of Engineering and \\ Information Technology, 64200, Rahim Yar Khan, Pakistan. \\ ${ }^{3}$ Centre for Advanced Studies in Pure and Applied Mathematics, Bahauddin Zakariya \\ University, 60800, Multan, Pakistan. \\ Email: ${ }^{1}$ shazib.hameed@kfueit.edu.pk, ${ }^{2}$ zaheeir@gmail.com, ${ }^{3}$ faisalali@bzu.edu.pk \\ Corresponding author: shazib.hameed@kfueit.edu.pk
}

Received: 26 April, 2021 / Accepted: 12 July, 2021 / Published online: 27 July, 2021

\begin{abstract}
We use the homotopy perturbation method (HPM) to construct a new iterative system for solving non-linear equations in this article. The criteria for convergence in the scheme developed are also imposed. To show the validity and reliability of our process, we compare our regime with other current procedures by looking at various test problems.
\end{abstract}

\section{AMS (MOS) Subject Classification Codes: 65H04, 65H05, 65H20}

Key Words: Iterative technique, Non-linear equations, Homotopy perturbation method, Two-step iterative technique, Order of convergence.

\section{INTRODUCTION}

We see multiple non-linear events in our daily lives. For many experts in science and technology, these phenomena are of considerable concern. Researchers have been focused on these approximate solutions to non-linear problems and during the last few decades, analytical solutions to these problems have been extensively studied. The researchers aimed at designing iterative methods with a high convergence order and requiring fewer calculations [7, 1, 3, 17], some work for the numerical solutions of different daily life problems using the homotopy perturbation method is published in recent years $[9,18,5,15,2,6]$. For numerical solutions of non-linear equations, He [10], Rafiq and Rafiullah [19], Golbabai and Javidi [7, 8], Noor and Noor [16], and Basto et al. [3] presented various techniques. Consider non-linear equation

$$
g(s)=0, \quad s \in R .
$$

In the fixed point iterative method for solving Eq. ( 1.1 ), we rearrange this equation as

$$
s=\hbar(s) \text {. }
$$

where

(i) $\exists[\alpha, \beta]$ s.t. $\forall s \in[\alpha, \beta] ; \hbar(s) \in[\alpha, \beta]$, 
(ii) $\exists l>0$ s.t. $\forall s \in[\alpha, \beta] ;\left|\hbar^{\prime}(s)\right| \leq l<1$.

Considering the following iterative scheme:

$$
s_{n+1}=\hbar\left(s_{n}\right), n=0,1,2,3, \ldots,
$$

and starting with a suitable initial approximation $s_{0}$, we build up a sequence of approximations, say $s_{n}$, for the solution of the non-linear equation, say $\rho$. The scheme will converge to the root $\rho$, provided that

(i) the initial approximation $s_{0}$ is chosen in the interval $[\alpha, \beta]$,

(ii) $\hbar$ has a continuous derivative on $(\alpha, \beta)$,

(iii) $\left|\hbar^{\prime}(s)\right|<1 \forall s \in[\alpha, \beta]$,

(iv) $\alpha<\hbar(s)<\beta \forall s \in[\alpha, \beta]$.

Consider the Taylor expansion of $\hbar\left(s_{n}\right)$ to calculate the order of convergence of the sequence $s_{0}$ :

$$
\hbar\left(s_{n}\right)=\hbar(s)+\left(s_{n}-s\right) \frac{\hbar^{\prime}(s)}{(1 !)}+\left(s_{n}-s\right)^{2} \frac{\hbar^{\prime \prime}(s)}{(2 !)}+\ldots+\left(s_{n}-s\right)^{k} \frac{\hbar^{(k)}(s)}{(k !)}+\ldots
$$

Then equations ( 1.2$),(1.3)$ and ( 1.4 ) yields

$$
s_{n+1}-s=\left(s_{n}-s\right) \hbar^{\prime}(s)+\left(s_{n}-s\right)^{2} \frac{\hbar^{\prime \prime}(s)}{(2 !)}+\ldots+\left(s_{n}-s\right)^{k} \frac{\hbar^{(k)}(s)}{(k !)}+\ldots,
$$

For the series of approximations deduced from an iterative process the order of convergence is characterized as:

Definition 1.1. Let $s_{0}, s_{1}, s_{2}, \ldots, s_{n}, \ldots$ be a succession of numerical approximations to a root $\rho$ i.e. $\lim _{n \rightarrow \infty} s_{n}=\rho$. Consider error at $n^{\text {th }}$ and $(n+1)^{\text {st }}$ steps be $e_{n}$ and $e_{n+1}$ respectively. and if

$\lim _{n \rightarrow \infty} \frac{\left|e_{n+1}\right|}{\left|e_{n}\right|^{\lambda}}=C$ for some $\lambda$ and non-zero constant $C$, then $\lambda$ is called convergence order of the method.

Definition 1.2. The iterative method has efficiency index amount $\lambda^{\frac{1}{n}}$ if the iterative method has a convergence order of $\lambda$ with an evaluations number of $n$ in the same iteration.

We state the following theorems.

Theorem 1.3. [20] Assume $g$ on $[\alpha, \beta]$ be continuous and in $(\alpha, \beta)$ be differentiable. Then $\exists$ a point $\xi \in(\alpha, \beta)$ such that

$$
g(t)=g(s)+(t-s) g^{\prime}(s)+\frac{1}{2}(t-s)^{2} g^{\prime \prime}(\xi) .
$$

Theorem 1.4. [4] Suppose $\hbar \in C^{p}[\alpha, \beta]$. If $\hbar^{\prime}(s)=\hbar^{\prime \prime}(s)=\ldots=\hbar^{(k-1)}(s)=0$ and $\hbar^{(k)}(s) \neq 0$, then sequence $s_{n}$ of order $k$.

\section{MODIFIED HPM}

He [11] set up the HPM in 1999 to deduce linear and non-linear problems. A coupling of perturbation method has also been presented by He [12]. HPM is therefore a composition of the method of homotopy and perturbation. The main purpose of the HPM is the 
uncomplicated and straightforward analysis of the current, complicated situation. Assume algebraic equations that are non-linear.

$$
g(s)=0, s \in R
$$

We claim that $\rho$ is a zero Eq. (2.6) and $\zeta$ is an initial estimate that is nearly to $\rho$. Using Theorem 1.3. about $\zeta$ Eq. ( 2. 6 ) implies

$$
g(\zeta)+g^{\prime}(\zeta)(s-\zeta)+g^{\prime \prime}(\xi) \frac{1}{2}(s-\zeta)^{2}=0
$$

Where $\xi \in(s, \zeta)$. We may re-write Eq. ( 2. 7 ) as

$$
s=c_{1}+M(s),
$$

where

$$
c_{1}=\zeta-\frac{g(\zeta)}{g^{\prime}(\zeta)},
$$

and

$$
M(s)=-\frac{1}{2}(s-\zeta)^{2} \frac{g^{\prime \prime}(\xi)}{g^{\prime}(\zeta)} .
$$

For Eq. ( 2.8 ), we build a homotopy $\Omega:(R \times[0,1]) \times R \rightarrow R$, to demonstrate basic concepts of the modified method of homotopy perturbation that satisfies

$$
\Omega(\omega, \epsilon, \vartheta)=\omega-c_{1}-\epsilon M(\omega)+\epsilon^{2}(1-\epsilon) \vartheta=0, \vartheta, \omega \in R, \epsilon \in[0,1],
$$

where $\vartheta$ be any real number, and $\epsilon$ be embedding parameter.

We see that

$$
\begin{gathered}
\Omega(\omega, 0, \vartheta)=\omega-c_{1}=0, \text { and } \\
\Omega(\omega, 1, \vartheta)=\omega-c_{1}-M(\omega)=0 .
\end{gathered}
$$

The simple problem $\Omega(\omega, 0, \vartheta)=\omega-c_{1}=0$ is constantly deformed into the original problem $\Omega(\omega, 1, \vartheta)=\omega-c_{1}-M(\omega)=0$ as $\epsilon$ monotonically increases from 0 to 1 . It has been earlier shown in literature by He in [11] that embedding parameter $\epsilon$ is used as an expanding parameter.

$$
\omega=s_{0}+\epsilon s_{1}+\epsilon^{2} s_{2}+\ldots
$$

The estimated solution to $\mathrm{Eq}$ ( 2. 6 ) is thus conveniently seen:

$$
\rho=\lim _{\epsilon \rightarrow 1} \omega=s_{0}+s_{1}+s_{2}+\ldots
$$

He proved convergence of the Series ( 2.15) in [13]. Using Taylor series of $M(\omega)$ about $s_{0}$ and then by applying our homotopy to Eq. ( 2. 6 ), Eq. ( 2. 11 ) $\Rightarrow$ :

$$
\begin{gathered}
\omega-c_{1}-\epsilon M\left(s_{o}\right)+\left(\omega-s_{0}\right) \frac{M^{\prime}\left(s_{0}\right)}{1 !}+ \\
\left(\omega-s_{0}\right)^{2} \frac{M^{\prime \prime}\left(s_{0}\right)}{2 !}+\ldots+\epsilon^{2}(1-\epsilon) \vartheta=0 .
\end{gathered}
$$


Using Eq. ( 2. 14 ) into Eq. ( 2. 16 ), We got

$$
\begin{aligned}
s_{0}+\epsilon s_{1}+\epsilon^{2} s_{2} & +\ldots-c_{1}-\epsilon\left\{M\left(s_{0}\right)+\left(s_{0}+\epsilon s_{1}+\epsilon^{2} s_{2}+\ldots-s_{0}\right) \frac{M^{\prime}\left(s_{0}\right)}{1 !}+\right. \\
& \left.\left(s_{0}+\epsilon s_{1}+\epsilon^{2} s_{2}+\ldots-s_{0}\right)^{2} \frac{M^{\prime \prime}\left(s_{0}\right)}{2 !}+\ldots\right\}+\epsilon^{2}(1-\epsilon) \vartheta=0 .(2.17)
\end{aligned}
$$

We can see by matching the terms with the equal power of the $\epsilon$ 's

$$
\begin{gathered}
\epsilon^{0}=s_{0}-c_{1}=0, \\
\epsilon^{1}=s_{1}-M\left(s_{0}\right)=0, \\
\epsilon^{2}=s_{2}-s_{1} M^{\prime}\left(s_{0}\right)+\vartheta=0, \\
\epsilon^{3}=s_{3}-s_{2} M^{\prime}\left(s_{0}\right)-\frac{1}{2} s_{1}^{2} M^{\prime \prime}\left(s_{0}\right)-\vartheta=0 .
\end{gathered}
$$

We're trying to find a $\vartheta$ in such a way that

$$
s_{2}=0
$$

Using $s_{1}=M\left(s_{0}\right)$ from Eq. ( 2. 19 ) into Eq. ( 2. 20 ), we have

$$
s_{2}-M\left(s_{0}\right) M^{\prime}\left(s_{0}\right)+\vartheta=0 .
$$

Since $s_{2}=0$ into Eq. (2.23), so that

$$
\vartheta=M\left(s_{0}\right) M^{\prime}\left(s_{0}\right) \text {. }
$$

Using Eq. ( 2. 24 ), $s_{1}=M\left(s_{0}\right)$, and $s_{2}=0$ into Eq. ( 2. 21 ), we have

$$
s_{3}=\frac{1}{2} M^{2}\left(s_{0}\right) M^{\prime \prime}\left(s_{0}\right)+M\left(s_{0}\right) M^{\prime}\left(s_{0}\right),
$$

where

$$
\begin{gathered}
M\left(s_{0}\right)=-\frac{g^{2}(\zeta) g^{\prime \prime}(\xi)}{2 g^{\prime 3}(\zeta)}, \\
M^{\prime}\left(s_{0}\right)=\frac{g(\zeta) g^{\prime \prime}(\xi)}{g^{\prime 2}(\zeta)}, \\
M^{\prime \prime}\left(s_{0}\right)=-\frac{g^{\prime \prime}(\xi)}{g^{\prime}(\zeta)}
\end{gathered}
$$

By comparing Eq. ( 2.9) and Eq. ( 2.18), we find

$$
s_{0}=c_{1}=\zeta-\frac{g(\zeta)}{g^{\prime}(\zeta)} .
$$

Using equations( 2. 26 )-( 2. 28 ) in Eq. ( 2. 19 ) and Eq. ( 2. 25 ), we have

$$
s_{1}=-\frac{g^{2}(\zeta) g^{\prime \prime}(\xi)}{2 g^{\prime 3}(\zeta)},
$$


and

$$
s_{3}=-\frac{g^{4}(\zeta) g^{\prime \prime 3}(\zeta)+4 g^{3}(\zeta) g^{\prime 2}(\zeta) g^{\prime \prime 2}(\xi)}{8 g^{7}(\zeta)}
$$

Manipulate equations( 2. 22 ), ( 2. 29 ), ( 2. 30 ) and ( 2. 31 ) into Eq. ( 2. 15 ), The solution of Eq. ( 2. 6 ) is found like as:

$$
\begin{gathered}
\rho=s_{0}+s_{1}+s_{2}+s_{3}+\ldots= \\
\zeta-\frac{g(\zeta)}{g^{\prime}(\zeta)}-\frac{g^{2}(\zeta) g^{\prime \prime}(\xi)}{2 g^{\prime 3}(\zeta)}-\frac{g^{4}(\zeta) g^{\prime \prime 3}(\zeta)+4 g^{3}(\zeta) g^{\prime 2}(\zeta) g^{\prime \prime 2}(\xi)}{8 g^{\prime 7}(\zeta)}+\ldots
\end{gathered}
$$

The aforementioned strategy for non-linear resolving Eq. ( 2. 6 ) helps us to propose these formulations.
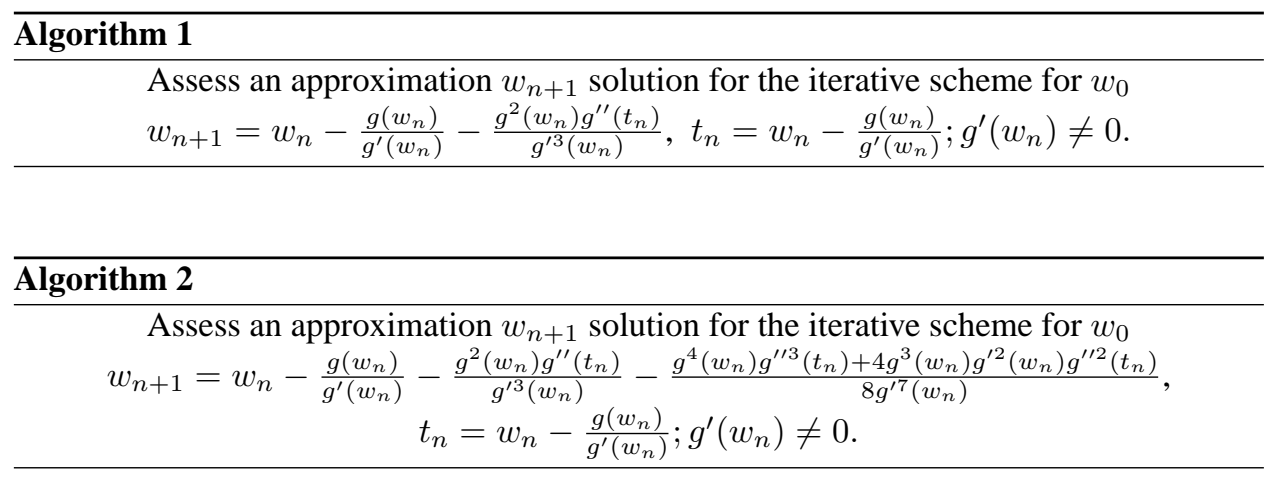

\section{CONVERGEnce AnAlysis}

The convergence of the Algorithm 2 is being explored.

Theorem 3.1. Identify the non-linear equation $g(s)=0$. Suppose that $g$ is sufficiently differentiable. If the initial guess $s_{0} \in[\alpha, \beta]$ close to the real root, then the convergence is at least order 3 for the Algorithm 2.

Proof. Assume $\rho$ be a fixed point of an iterative function, as defined in the Algorithm 2, let us define

$$
t=s-\frac{g(s)}{g^{\prime}(s)}
$$

Now consider

$$
\hbar(s)=s-\frac{g(s)}{g^{\prime}(s)}-\frac{[g(s)]^{2} g^{\prime \prime}(t)}{2\left[g^{\prime}(s)\right]^{3}}-\frac{4[g(s)]^{3}\left[g^{\prime}(s)\right]^{2}\left[g^{\prime \prime}(t)\right]^{2}+[g(s)]^{4}\left[g^{\prime \prime}(t)\right]^{3}}{8\left[g^{\prime}(s)\right]^{7}} .
$$

With Mathematica tools, derivatives and derivative values of $\hbar(s)$ at $\rho$ can be easily accessed as follows:

$$
\begin{array}{r}
\hbar(\rho)=\rho, \hbar^{\prime}(\rho)=0=\hbar^{\prime \prime}(\rho), \text { but } \\
\hbar^{\prime \prime \prime}(\rho)=\frac{6\left[g^{\prime \prime}(\rho)\right]^{2}}{\left[g^{\prime}(\rho)\right]^{2}}-\frac{g^{(3)}(\rho)}{g^{\prime}(\rho)}-3 g^{\prime}(\rho)\left(\frac{2\left[g^{\prime \prime}(\rho)\right]^{2}}{\left[g^{\prime}(\rho)\right]^{3}}-\frac{g^{(3)}(\rho)}{\left[g^{\prime}(\rho)\right]^{2}}\right) .
\end{array}
$$


Obviously $\hbar^{\prime \prime \prime}(\rho) \neq 0$ implies $\frac{6\left[g^{\prime \prime}(\rho)\right]^{2}}{\left[g^{\prime}(\rho)\right]^{2}}-\frac{g^{(3)}(\rho)}{g^{\prime}(\rho)}-3 g^{\prime}(\rho)\left(\frac{2\left[g^{\prime \prime}(\rho)\right]^{2}}{\left[g^{\prime}(\rho)\right]^{3}}-\frac{g^{(3)}(\rho)}{\left[g^{\prime}(\rho)\right]^{2}}\right) \neq 0$.

The aforementioned equation shows that the convergence order of the Algorithm is 3 .

Also, our Algorithm 2 has a number of evaluations at each step is 3, So the efficiency index of our proposed method is $3^{\frac{1}{3}}=1.44225$.

Note: The Newton Raphson method is quadratically convergent and the method of Javidi, Noor method, and Rafiq and Rafiullah are cubically convergent. The number of evaluations at each step of the Newton Raphson method is 2, the method of Javidi is 4, the Noor method, and Rafiq and Rafiullah both are 3. Similarly, the efficiency index of the Newton Raphson method is 1.41421 , the method of Javidi is 1.31607 , the Noor method, and Rafiq and Rafiullah both are 1.44225 .

\section{TEST PROBLEMS}

We give several examples in this section to demonstrate the applicability of Algorithm 2 (ALGO2) developed in the previous section. For each problem in a table format, the comparison of our method i.e. the Algorithm 2 (ALGO2) with the method of Javidi (JM) Algorithm 2.3 [14], Newton-Raphson method (NR), the Noor (NM) method Algorithm 2 [16], and the Rafiq and Rafiullah method (RR) Algorithm 2 [19] is given. To calculate the outcomes, Maple software was used.

Example 4.1. Non-linear equation $s-2-e^{(-s)}=0$ with initial guess $s_{0}=-1.5$ and $s=2.12002823898764122948$ is the precise prospected solution.

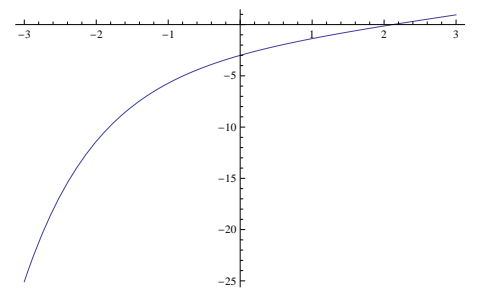

FIGURE 1. Graph of Example 1

TABLE 1. Approximate consequences for Example 1

\begin{tabular}{|c|c|c|c|c|}
\hline Method & Number of iterations & Evaluation & $s[k]$ & $|g(s[k])|$ \\
\hline$N R$ & 6 & 12 & 2.1200282389876412 & $3.302368 e-17$ \\
\hline$J M$ & Diverges & - & - & - \\
\hline$N M$ & 5 & 15 & 2.1200282389876412 & $3.302368 e-17$ \\
\hline$R R$ & 5 & 15 & 2.1200282389876412 & $3.302368 e-17$ \\
\hline$A L G O 2$ & 5 & 15 & 2.1200282389876412 & $3.302368 e-17$ \\
\hline
\end{tabular}

Example 4.2. Equation $(s-1)^{20}-2 s+2=0$ with $s_{0}=0.2$ and $s=1.0000000000000000$ is the precise prospected solution. 


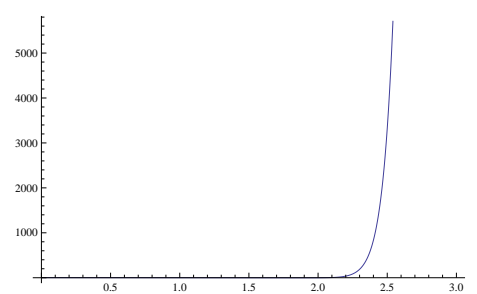

FIGURE 2. Graph of Example 2

TABLE 2. Approximate consequences for Example 2

\begin{tabular}{|c|c|c|c|c|}
\hline Method & Number of iterations & Evaluation & $s[k]$ & $|g(s[k])|$ \\
\hline$N R$ & 2 & 4 & 1.0000000000000000 & $0.000000 e+00$ \\
\hline$J M$ & Diverges & - & - & - \\
\hline$N M$ & 2 & 6 & 1.0000000000000000 & $0.000000 e+00$ \\
\hline$R R$ & 2 & 6 & 1.0000000000000000 & $0.000000 e+00$ \\
\hline$A L G O 2$ & 2 & 6 & 1.0000000000000000 & $0.000000 e+00$ \\
\hline
\end{tabular}

Example 4.3. Non-linear equation $e^{s}-3 s^{2}=0$ with initial guess $s_{0}=1.5$ and $s=$ 0.91000757248870906066 is the precise prospected solution.

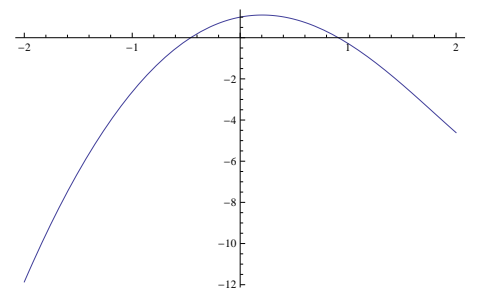

FIGURE 3. Graph of Example 3

TABLE 3. Approximate consequences for Example 3

\begin{tabular}{|c|c|c|c|c|}
\hline Method & Number of iterations & Evaluation & $s[k]$ & $|g(s[k])|$ \\
\hline$N R$ & 5 & 10 & 0.9100075724887091 & $1.956044 e-18$ \\
\hline$J M$ & 3 & 12 & 0.9100075724887091 & $3.171309 e-17$ \\
\hline$N M$ & 3 & 9 & 0.9100075724887091 & $1.956044 e-18$ \\
\hline$R R$ & 4 & 12 & 0.9100075724887091 & $1.956044 e-18$ \\
\hline$A L G O 2$ & 3 & 9 & 0.9100075724887091 & $1.956044 e-18$ \\
\hline
\end{tabular}

Example 4.4. Non-linear equation $\ln (s)=0$ with initial guess $s_{0}=0.4$ and 1.00000000000000000000 is the precise prospected solution. 


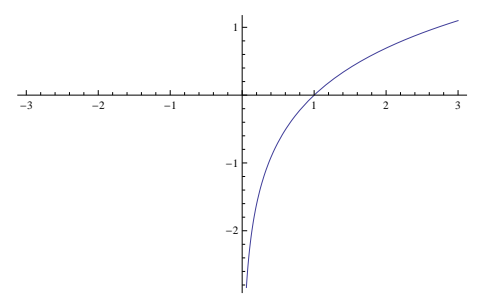

FIGURE 4. Graph of Example 4

TABLE 4. Approximate consequences for Example 4

\begin{tabular}{|c|c|c|c|c|}
\hline Method & Number of iterations & Evaluation & $s[k]$ & $|g(s[k])|$ \\
\hline$N R$ & 5 & 10 & 0.9999999999999951 & $4.880000 e-15$ \\
\hline$J M$ & 5 & 20 & 1.0000000000000000 & $0.000000 e+00$ \\
\hline$N M$ & 4 & 12 & 1.0000000000000000 & $0.000000 e+00$ \\
\hline$R R$ & 4 & 12 & 1.0000000000000000 & $0.000000 e+00$ \\
\hline$A L G O 2$ & 4 & 12 & 1.0000000000000000 & $0.000000 e+00$ \\
\hline
\end{tabular}

Example 4.5. Non-linear equation $s^{5}+s^{4}+4 s^{2}-15=0$ with initial guess $s_{0}=1.5$ and $s=1.34742809896830498151$ is the precise prospected solution.

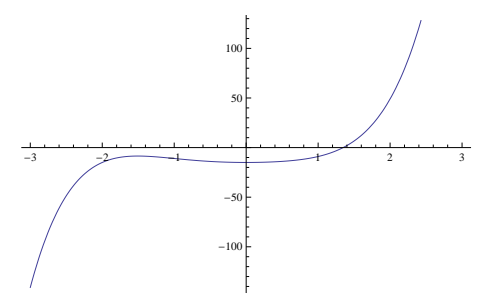

FIGURE 5. Graph of Example 5

TABLE 5. Approximate consequences for Example 5

\begin{tabular}{|c|c|c|c|c|}
\hline Method & Number of iterations & Evaluation & $s[k]$ & $|g(s[k])|$ \\
\hline$N R$ & 5 & 10 & 1.3474280989683050 & $6.851044 e-16$ \\
\hline$J M$ & 3 & 12 & 1.3474280989683050 & $6.851044 e-16$ \\
\hline$N M$ & 3 & 9 & 1.3474280989683050 & $6.851044 e-16$ \\
\hline$R R$ & 3 & 9 & 1.3474280989683050 & $6.851044 e-16$ \\
\hline$A L G O 2$ & 3 & 9 & 1.3474280989683050 & $6.851044 e-16$ \\
\hline
\end{tabular}

Example 4.6. Equation $\sin (s)-\frac{s}{3}=0$ with $s_{0}=1.9$. 


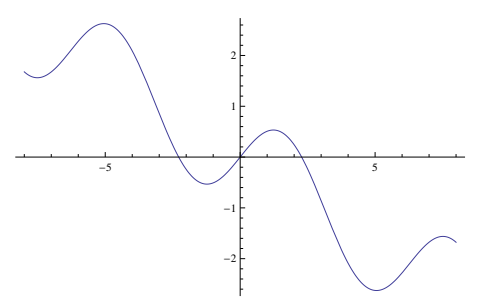

FIGURE 6. Graph of Example 6

TABLE 6. Approximate consequences for Example 6

\begin{tabular}{|c|c|c|c|c|}
\hline Method & Number of iterations & Evaluation & $s[k]$ & $|g(s[k])|$ \\
\hline$N R$ & 5 & 10 & 2.2788626600758283 & $1.249294 e-17$ \\
\hline$J M$ & 4 & 16 & 2.2788626600758283 & $1.249294 e-17$ \\
\hline$N M$ & 3 & 9 & 2.2788626600758283 & $1.249294 e-17$ \\
\hline$R R$ & 3 & 9 & 2.2788626600758283 & $1.249294 e-17$ \\
\hline ALGO2 & 3 & 9 & 2.2788626600758283 & $1.249294 e-17$ \\
\hline
\end{tabular}

Example 4.7. $10 s e^{-s^{2}}-1=0$ with $s_{0}=0$, and $s=0.10102584831568519737$ is the precise prospected solution.

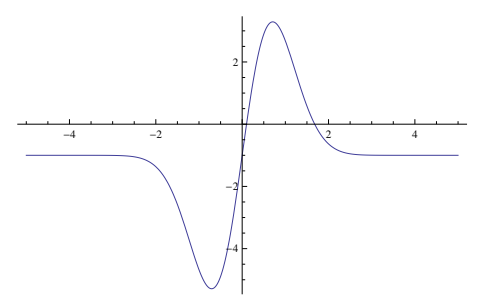

FIGURE 7. Graph of Example 7

TABLE 7. Approximate consequences for Example 7

\begin{tabular}{|c|c|c|c|c|}
\hline Method & Number of iterations & Evaluation & $s[k]$ & $|g(s[k])|$ \\
\hline$N R$ & 4 & 8 & 0.1010258483156852 & $2.552517 e-17$ \\
\hline$J M$ & 3 & 12 & 0.1010258483156852 & $2.552517 e-17$ \\
\hline$N M$ & 3 & 9 & 0.1010258483156852 & $2.552517 e-17$ \\
\hline$R R$ & 3 & 9 & 0.1010258483156852 & $2.552517 e-17$ \\
\hline ALGO2 & 3 & 9 & 0.1010258483156852 & $2.552517 e-17$ \\
\hline
\end{tabular}

Example 4.8. $e^{-s^{2}+s+2}-1=0$ with $s_{0}=2.1$, where $s=2.00000000000000000000$ is the precise prospected solution. 


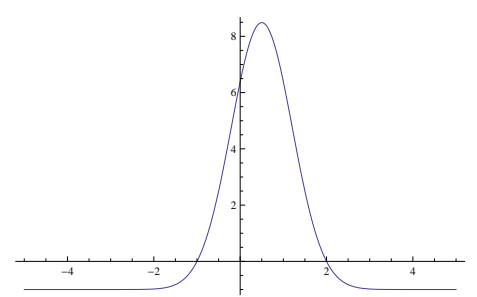

FIGURE 8. Graph of Example 8

TABLE 8. Approximate consequences for Example 8

\begin{tabular}{|c|c|c|c|c|}
\hline Method & Number of iterations & Evaluation & $s[k]$ & $|g(s[k])|$ \\
\hline$N R$ & 4 & 8 & 1.9999999999999969 & $9.300000 e-15$ \\
\hline$J M$ & 3 & 12 & 2.0000000000000001 & $3.000000 e-16$ \\
\hline$N M$ & 3 & 9 & 2.0000000000000000 & $0.000000 e+00$ \\
\hline$R R$ & 3 & 9 & 2.0000000000000000 & $0.000000 e+00$ \\
\hline$A L G O 2$ & 3 & 9 & 2.0000000000000000 & $0.000000+00$ \\
\hline
\end{tabular}

Example 4.9. $\ln \left(s^{2}+s+2\right)-s+1=0$ with $s_{0}=-1$ and $s=4.15259073675715827500$ is the precise prospected solution.

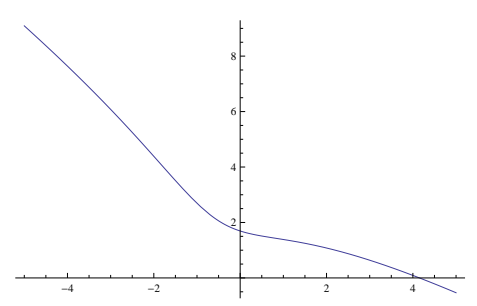

FIGURE 9. Graph of Example 9

TABLE 9. Approximate consequences for Example 9

\begin{tabular}{|c|c|c|c|c|}
\hline Method & Number of iterations & Evaluation & $s[k]$ & $|g(s[k])|$ \\
\hline$N R$ & 6 & 12 & 4.1525907367571629 & $2.785569 e-15$ \\
\hline$J M$ & - & diverges & - & - \\
\hline$N M$ & 5 & 15 & 4.1525907367571583 & $1.505894 e-17$ \\
\hline$R R$ & 5 & 15 & 4.1525907367571583 & $1.505894 e-17$ \\
\hline ALGO2 & 5 & 15 & 4.1525907367571583 & $1.505894 e-17$ \\
\hline
\end{tabular}

Example 4.10. $\cos (s)-s=0$ with $s_{0}=1$ and $s=0.73908513321516064166$ is the precise prospected solution. 


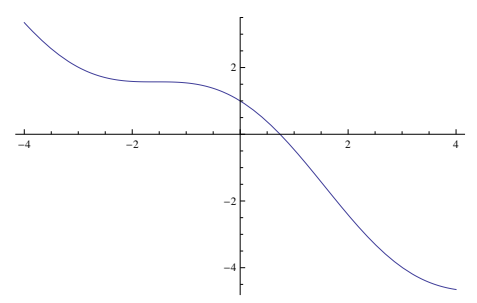

FIGURE 10. Graph of Example 10

TABLE 10. Approximate consequences for Example 10

\begin{tabular}{|c|c|c|c|c|}
\hline Method & Number of iterations & Evaluation & $s[k]$ & $|g(s[k])|$ \\
\hline$N R$ & 4 & 8 & 0.7390851332151606 & $2.770350 e-18$ \\
\hline$J M$ & 3 & 12 & 0.7390851332151606 & $2.770350 e-18$ \\
\hline$N M$ & 3 & 9 & 0.7390851332151606 & $2.770350 e-18$ \\
\hline$R R$ & 3 & 9 & 0.7390851332151606 & $2.770350 e-18$ \\
\hline ALGO2 & 3 & 9 & 0.7390851332151606 & $2.770350 e-18$ \\
\hline
\end{tabular}

Example 4.11. $e^{-s}+\cos (s)=0$ with $s_{0}=1.75$ and $s=1.74613953040801241765$ is the precise prospected solution.

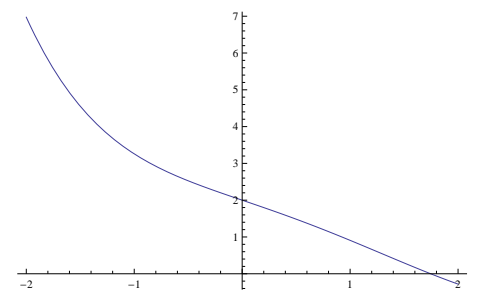

FIGURE 11. Graph of Example 11

TABLE 11. Approximate consequences for Example 11

\begin{tabular}{|c|c|c|c|c|}
\hline Method & Number of iterations & Evaluation & $s[k]$ & $|g(s[k])|$ \\
\hline$N R$ & 3 & 6 & 1.7461395304080124 & $2.045916 e-17$ \\
\hline$J M$ & 2 & 8 & 1.7461395304080124 & $2.045916 e-17$ \\
\hline$N M$ & 2 & 6 & 1.7461395304080124 & $2.045916 e-17$ \\
\hline$R R$ & 2 & 6 & 1.7461395304080124 & $2.045916 e-17$ \\
\hline ALGO2 & 2 & 6 & 1.7461395304080124 & $2.045916 e-17$ \\
\hline
\end{tabular}

Example 4.12. $\sin ^{-1}\left(s^{2}-1\right)-\frac{s}{2}+1=0$ with $s_{0}=0.3$ and $s=0.59481096839836917752$ is the precise prospected solution. 


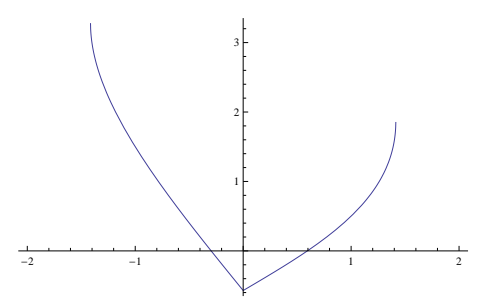

FIGURE 12. Graph of Example 12

TABLE 12. Approximate consequences for Example 12

\begin{tabular}{|c|c|c|c|c|}
\hline Method & Number of iterations & Evaluation & $s[k]$ & $|g(s[k])|$ \\
\hline$N R$ & 4 & 8 & 0.5948109683983692 & $2.622997 e-18$ \\
\hline$J M$ & 3 & 12 & 0.5948109683983692 & $2.622997 e-18$ \\
\hline$N M$ & 3 & 9 & 0.5948109683983692 & $7.964943 e-18$ \\
\hline$R R$ & 3 & 9 & 0.5948109683983692 & $2.622997 e-18$ \\
\hline ALGO2 & 3 & 9 & 0.5948109683983692 & $7.964943 e-18$ \\
\hline
\end{tabular}

\section{CONCLUSION}

A new computational strategy based on the HPM was developed for the settlement of non-linear algebraic equations. The method created in this paper is also compared with the method of Newton-Raphson (NR), the Javidi method (JM) [14], the Noor method (NM) [16] and Rafiq and Rafiullah's method (RR) [19]. The findings behind the work contained in the current paper are the motivation retrieved from Rafiq and Rafiullah [19]. Depending on the approach of HPM, we have explored a new scheme for evaluating non-linear equations. The technique developed in this paper is a two-step predictor-corrector method. In determining the effectiveness of a numerical method, the efficiency index is a key parameter. The efficiency index of our proposed methods is, 1.44225 , which is better or equal to different methods which are well-known.

Some problems are suggested in the last section of this paper in which our method compared with various established popular methods. The outcomes of these problems indicate the efficiency and validity of the success of our strategies.

\section{ACKNOWLEDGMENTS}

The authors express their sincere gratitude to the Editor and referees for the careful reading of the original manuscript and useful comments that helped to improve the presentation of the results.

Authors Contributions: The third author gave the idea of the main results. All the authors contributed equally to the writing of this paper. All the authors read and approved the final manuscript.

Competing interest: The authors declare that they have no competing interest regarding this research work. 


\section{REFERENCES}

1. S. Abbasbandy, Improving Newton-Raphson method for nonlinear equations by modified Adomian decomposition method, Appl. Math. Comput. 145, (2003) 887-893.

2. T. G. Ashrafi, S. Hoseinzadeh, A. Sohani, M. H. Shahverdian, Applying homotopy perturbation method to provide an analytical solution for Newtonian fluid flow on a porous flat plate, Mathematical Methods in the Applied Sciences, 44, No. 8 ((2021)) 7017-7030.

3. M. Basto, V. Semiao, F. L. Calheiros, A new iterative method to compute nonlinear equations, Appl. Math. Comput. 173, (2006) 468-483.

4. E. Babolian, J. Biazar, On the Order of Convergence of Adomian Method, Journal of Applied Mathematics and Computation 130, (2002) 383387.

5. H. Dehghani, I. Mansouri, A. Farzampour, J. W. Hu, Improved homotopy perturbation method for geometrically nonlinear analysis of space trusses, Applied Sciences, 10, No. 8 (2020) 2987.

6. Y. O. El-Dib, Homotopy perturbation method with rank upgrading technique for the superior nonlinear oscillation, Mathematics and Computers in Simulation, 182, (2021) 555-565.

7. A. Golbabai, M. Javidi, A third-order Newton type method for nonlinear equations based on modified homotopy perturbation method, Appl. Math. Comput. 191, (2007) 199-205.

8. A. Golbabai, M. Javidi, A new family of iterative methods for solving system of nonlinear algebric equations, Appl. Math. Comput. 190, No. 2 (2007) 1717-1722.

9. N. Gupta, N. Kanth, Analytical approximate solution of heat conduction equation using new homotopy perturbation method, Matrix Science Mathematic (MSMK), 3, No. 2 (2019) 01-07.

10. J. H. He, A new iterative method for solving algebric equations, Appl. Math. Comput. 135, (2003) 81-84.

11. J. H. He, Homotopy perturbation technique, Comput. Methods Appl. Mech. Eng. 178, No. (3-4) (1999) 257-262.

12. J. H. He, A coupling method of a homotopy technique and a perturbation technique for non-linear problems, Int. J. Non-Linear Mech. 35 No.1 (2000) 37-43.

13. J. H. He, Homotopy perturbation technique, Comput. Methods Appl. Mech. Eng. 178, No. (3-4) (1999) 257-262.

14. M. Javidi, Iterative methods to nonlinear equations, Appl. Math. Comput. 193, (2007) 360-365.

15. M. J. Mahmoodabadi, Epidemic model analyzed via particle swarm optimization based homotopy perturbation method, Informatics in Medicine Unlocked, 18, (2020) 100293.

16. M. A. Noor, K. I. Noor, Modified iterative methods with cubic convergence for solving nonlinear equations, Appl. Math. Comput. 184, No. 2 (2007) 322-325.

17. A.Y. Özban, Some new variants of Newton's method, Appl. Math. Lett. 17, (2004) 677-682.

18. S. A. Pasha, Y. Nawaz, M. S. Arif, The modified homotopy perturbation method with an auxiliary term for the nonlinear oscillator with discontinuity, Journal of Low Frequency Noise, Vibration and Active Control, 38, No. (3-4) (2019) 1363-1373.

19. A. Rafiq, M. Rafiullah, Some multi-step iterative methods for solving nonlinear equations, Comput. Math. Appl. 58, (2009) 1589-1597.

20. W. Rudin, Principles of Mathematical Analysis, McGraw-Hill Book Company, Inc., 1953. 